\title{
SNX3 suppresses the migration and invasion of colorectal cancer cells by reversing epithelial-to-mesenchymal transition via the $\beta$-catenin pathway
}

\author{
BIRAN PAN ${ }^{1}$, TONGTONG ZHANG ${ }^{1}$, WEI YANG ${ }^{1}$, YANJUN LIU $^{2}$, YUNING CHEN $^{3}$, ZHENG ZHOU $^{1}$, \\ YAN TANG $^{4}$, JIAWEI ZENG ${ }^{5}$, YILUN LIU ${ }^{6}, \mathrm{CONG} \mathrm{ZHAO}^{7}$ and YUANBIAO GUO $^{1}$
}

${ }^{1}$ Medical Research Center; ${ }^{2}$ Department of Gastrointestinal Surgery, The Third People's Hospital of Chengdu, The Second Chengdu Hospital Affiliated to Chongqing Medical University, The Affiliated Hospital of Southwest Jiaotong University, Chengdu, Sichuan 610036; ${ }^{3}$ Clinical Laboratory Department, Xindu People's Hospital, Chengdu, Sichuan 610500; ${ }^{4}$ Department of Pathology, The Third People's Hospital of Chengdu, The Second Chengdu Hospital Affiliated to Chongqing Medical University, The Affiliated Hospital of Southwest Jiaotong University, Chengdu,

Sichuan 610036; ${ }^{5}$ Clinical Laboratory Department, Mianyang Central Hospital, Mianyang, Sichuan 621000;

${ }^{6}$ Clinical Laboratory Department, The First Affiliated Hospital of Chengdu Medical College, Chengdu, Sichuan 610500; ${ }^{7}$ Department of Digestive Diseases, The Third People's Hospital of Chengdu, The Second Chengdu Hospital Affiliated to Chongqing Medical University, The Affiliated Hospital of Southwest Jiaotong University, Chengdu, Sichuan 610036, P.R. China

Received July 27, 2018; Accepted July 10, 2019

DOI: 10.3892/ol.2019.10860

\begin{abstract}
The $\mathrm{Wnt} / \beta$-catenin signaling pathway is a well-studied pathway that drives the carcinogenesis and metastasis of colorectal cancer (CRC). The secretion of Wnt proteins is essential for the continuous activation of Wnt/ $\beta$-catenin signaling in CRC. The secretion of Drosophila wingless, which is homologous to the human Wnt protein, is mediated by sorting nexin 3 (SNX3) in Drosophila; however, the role of SNX3 in CRC remains unknown. In the present study it was demonstrated that SNX3 reduced the migratory and invasive ability of HCT116 human CRC cells, and reversed epithelial-mesenchymal transition (EMT). Conversely, in the HT29 CRC cell line, which endogenously expresses high levels
\end{abstract}

Correspondence to: Dr Yuanbiao Guo, Medical Research Center, The Third People's Hospital of Chengdu, The Second Chengdu Hospital Affiliated to Chongqing Medical University, The Affiliated Hospital of Southwest Jiaotong University, 82 Qinglong Road, Chengdu, Sichuan 610036, P.R. China

E-mail: guo.ybiao@yahoo.com

Dr Cong Zhao, Department of Digestive Diseases, The Third People's Hospital of Chengdu, The Second Chengdu Hospital Affiliated to Chongqing Medical University, The Affiliated Hospital of Southwest Jiaotong University, 82 Qinglong Road, Chengdu, Sichuan 610036, P.R. China

E-mail: czhao0050@sohu.com

Key words: sorting nexin 3, colorectal cancer, metastases, $\beta$-catenin, epithelial-mesenchymal transition of SNX3, short hairpin RNA or siRNA-mediated knockdown of SNX3 induced EMT, and enhanced cell migration and invasion. In addition, upregulation of SNX3 significantly inhibited metastasis of HCT116 cells to the lungs of mice. These SNX3-mediated effects were associated with downregulation of $\beta$-catenin. Taken together, by downregulating $\beta$-catenin, SNX3 may mediate EMT and reverse CRC metastasis.

\section{Introduction}

Colorectal cancer (CRC) is the third most common type of cancer, accounting for 771,000 cases of mortality annually worldwide (1). Metastasis is the major cause of mortality in patients with CRC; however, effective control strategies are limited. In the past few decades, dysfunctional Wnt signaling has been demonstrated to be the major contributor to CRC tumorigenesis and metastasis (2). Over $90 \%$ of CRC cases carry mutations in genes encoding proteins involved in the $\mathrm{Wnt} / \beta$-catenin pathway (2). Therefore, understanding the effects of $\mathrm{Wnt} / \beta$-catenin signaling on the development and metastasis of CRC may be useful in identifying potential therapeutic targets (3).

In intestinal epithelial cells, excessive Wnt protein expression continuously activates Wnt/ $\beta$-catenin signaling and drives epithelial-mesenchymal transition (EMT) (4), which is considered to be one of the major determinants of metastasis (5). Conversely, inhibition of Wnt secretion reverses EMT of CRC cells (4). Sorting nexin 3 (SNX3) has been demonstrated to regulate the secretion of Drosophila Wingless (Wg) which is the homolog of human Wnt, via retromer-dependent Wntless recycling $(6,7)$. Therefore it has been hypothesized that human SNX3 may affect EMT or the metastases of CRC cells. In the present study, it was demonstrated that SNX3 inhibited $\beta$-catenin 
signaling in CRC cells and reversed the EMT process, thereby reducing the metastasis of $\mathrm{CRC}$ cells in vitro and in vivo.

\section{Materials and methods}

Cell culture. Human CRC cell lines (HCT116, HT29, SW480, SW620, SW1116, HCT8, RKO, Colo205, LOVO, Colo320DM and NCI-H716) and a human normal colon epithelial cell line, NCM460, were obtained from the Type Culture Collection of the Chinese Academy of Medical Sciences. All cells were maintained in RPMI-1640 medium (Invitrogen; Thermo Fisher Scientific Inc.), supplemented with $10 \%$ (v/v) fetal bovine serum (HyClone; GE Healthcare Life Sciences) in a humidified incubator at $37^{\circ} \mathrm{C}$ and $5 \% \mathrm{CO}_{2}$.

Virus infection, small interfering (si)RNA and small hairpin (sh)RNA transfection. SNX3 expression lentivirus and a negative control lentivirus were purchased from GeneCopoeia, Inc. HCT116 cells were seeded into 24-well plates at a density of $4 \times 10^{5}$ cells/well. The HCT116 cells were infected by removing the old culture medium and replacing it with $0.5 \mathrm{ml}$ diluted viral supernatant and incubated at the $37^{\circ} \mathrm{C}$ overnight. The clones with stable SNX3 expression were selected using $2 \mu \mathrm{g} / \mathrm{ml}$ puromycin for 2 weeks.

For the siRNA-SNX3 experiments, cells were seeded in 6 -well plates at a density of $1 \times 10^{5}$ cells/well and medium was replaced with serum-free medium once confluence reached $\sim 80 \%$. Subsequently, $6 \mu 1$ siRNA-SNX3 or negative control siRNA (NC siRNA) (Shanghai GenePharma Co., Ltd.) was mixed with $94 \mu$ l Opti-Minimum Essential Medium (Gibco; Thermo Fisher Scientific, Inc.) and $12 \mu 1$ HiPerfect transfection reagent (Qiagen China Co., Ltd.). The oligonucleotide sequences were as follows: siRNA-SNX3-01 sense strand, 5'-GCGUCAGCUUCCUUUUAGATT-3' and antisense strand, 5'-UCUAAAAGGAAGCUGACGCTT-3'; siRNA-SNX3-02 sense strand, 5'-CCAGCAACUUCCUCGAGAUTT-3' and antisense strand, 5'-AUCUCGAGGAAGUUGCUGGTT-3'; and NC siRNA sense strand, 5'-UUCUCCGAACGUGUC ACGUTT-3' and antisense strand, 5'-ACGUGACACGUU CGGAGAATT-3'. The mixture was gently agitated and subsequently incubated for 5-10 $\mathrm{min}$ at room temperature, added to the cells, and the cells were incubated for $48 \mathrm{~h}$ at $37^{\circ} \mathrm{C}$.

HT29 cells were seeded in 6-well plates at $2 \times 10^{5}$ cells/well. The following day, $1 \mu \mathrm{g}$ pLKO.1 puro plasmid (Addgene, Inc.) encoding either human SNX3 shRNA or NC shRNA were mixed with $3.75 \mu$ l HiPerfect transfection reagent (Qiagen China Co., Ltd.) and $150 \mu \mathrm{l}$ RPMI-1640 medium. The mixture was gently mixed and subsequently incubated for $10-15 \mathrm{~min}$ at room temperature, after which it was added to the cells. After $48 \mathrm{~h}$, transfected cells were selected using $2 \mu \mathrm{g} / \mathrm{ml}$ puromycin for 2 weeks. The medium was replaced once every three days. For the si- $\beta$-catenin experiments, the shSNX3-HT29 and shNC-HT29 cells were transfected with the siRNA- $\beta$-catenin or NC siRNA for $48 \mathrm{~h}$. $\beta$-catenin siRNA sense strand, 5 '-GCU GCUUUAUUCUCCCAUUTT-3' and antisense strand, 5'-AAUGGGAGAAUAAAGCAGCTT-3' were synthesized by Shanghai GenePharma Ltd.

Western blot analysis. Cells were lysed in RIPA buffer (Beyotime Institute of Biotechnology) supplemented with
Protease Inhibitor Cocktail (Roche Diagnostics). Equal quantities $(20 \mu \mathrm{g})$ of total protein were separated by SDS-PAGE using a $10 \%$ gel and subsequently transferred onto polyvinylidene fluoride membranes. The membranes were blocked with $5 \%$ skimmed milk and immunoblotted with the primary antibodies against $\beta$-catenin $(1: 1,000)$, E-cadherin $(1: 1,000)$, vimentin $(1: 1,000)$ cat. nos. 8480, 3195 and 5741 respectively; Cell Signaling Technology, Inc.), SNX3 (1:1,000; cat. no. ab56078; Abcam) and GAPDH (1:2,000; cat. no. 60004-1-Ig; ProteinTech Group, Inc.) at $4^{\circ} \mathrm{C}$ overnight; antibodies were diluted in 5\% BSA (Beijing Solarbio Science \& Technology Co., Ltd.) in Tris-buffered saline. Following primary antibody incubation, membranes were probed with anti-mouse immunoglobulin $\mathrm{G}(\mathrm{IgG})$ or anti-rabbit IgG secondary antibodies $(1: 10,000$; cat. no. SA00001-01 or SA00001-02, respectively; ProteinTech Group, Inc.) at the room temperature for $2 \mathrm{~h}$. The signal was visualized using Immobilon Western Chemiluminescent HRP Substrate (EMD Millipore) according to the manufacturer's protocol. The detection of GAPDH was used as loading control and for densitometric analysis. The intensity of the bands was semi-quantified using ImageJ version 1.46r (National Institutes of Health).

Immunocytochemistry. Cells were fixed in $4 \%$ paraformaldehyde at room temperature for $30 \mathrm{~min}$ and blocked with goat serum (cat. no. SL038; Solarbio Life Sciences, Inc.) for $1 \mathrm{~h}$ at $37^{\circ} \mathrm{C}$. Subsequently, cells were incubated with E-cadherin and vimentin antibodies (1:100; ProteinTech Group, Inc.) at $4^{\circ} \mathrm{C}$ overnight. The cells were washed with PBS and incubated with Alexa Fluor ${ }^{\circledR}$ 594-conjugated secondary antibodies (1:200; cat. no. A32740; Invitrogen; Thermo Fisher Scientific, Inc.) at 1:1,000 for $1 \mathrm{~h}$ at $37^{\circ} \mathrm{C}$. DAPI (Beijing Solarbio Science \& Technology Co., Ltd.) was used to stain the cell nuclei for $5 \mathrm{~min}$ at the room temperature. Images were captured using a fluorescence microscope (Carl Zeiss AG, Oberkochen, Germany).

Invasion and migration assays. Cell migration and invasion assays were performed using a 24-well migration chamber (Corning, Inc.) with or without Matrigel ${ }^{\mathrm{TM}}$, respectively. For the cell migration assays, cells at a density of $5 \times 10^{5}$ in $200 \mu \mathrm{l}$ serum-free medium were seeded onto Transwell inserts. The bottom chamber was filled with $600 \mu 1$ medium containing $20 \%$ FBS. For the invasion assays, Transwell inserts were coated with $25 \mu \mathrm{g}$ Matrige ${ }^{\mathrm{TM}}$. After incubation for $48 \mathrm{~h}$, the inserts were fixed with neat methanol at room temperature for $20 \mathrm{~min}$ and stained with $2 \%$ crystal violet for $30 \mathrm{sec}$ at the room temperature. The number of cells which had invaded through the membrane per field was counted and imaged under a light microscope (magnification, x200; Carl Zeiss AG).

Wound healing assay. The HT29 and HCT116 cells were seeded in 6-well plates at a density of $1 \times 10^{6}$ cells/well. A scratch was made in the center of the well using a sterile 100- $\mu 1$ pipette tip once the confluence reached $\sim 95 \%$. The cells were washed three times with PBS and the medium was replaced with fresh serum-free medium. Images were captured on an inverted light microscope (magnification, x100; Carl Zeiss AG) at 0 and $24 \mathrm{~h}$. Results were expressed as the migration index; the distance migrated by HT29 relative to the distance migrated by HCT116 (8). 
A

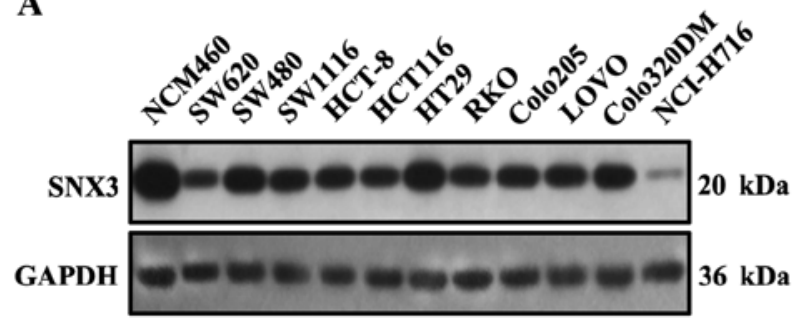

B

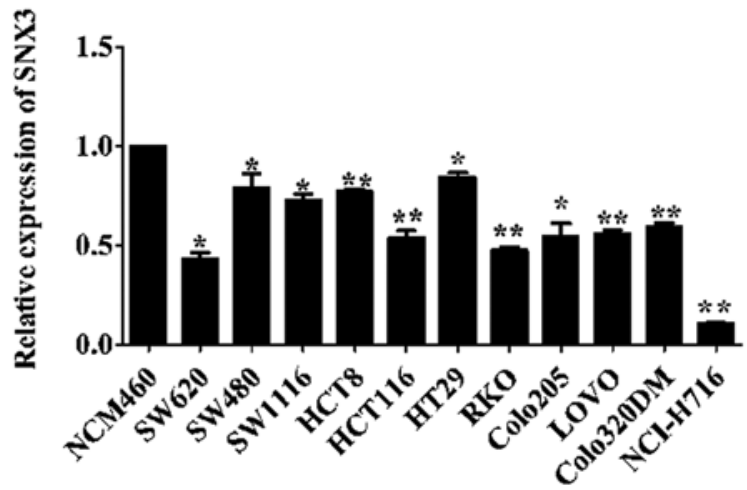

Figure 1. Endogenous SNX3 expression is downregulated in human CRC cells compared with in healthy colorectal cells. (A) Western blot analysis was performed to examine SNX3 expression in 11 CRC cell lines and a human normal colon epithelial cell line. (B) Densitometric analysis of SNX3 expression in the CRC cells relative to the normal colon epithelial cell line NCM460. ${ }^{*} \mathrm{P}<0.05,{ }^{* *} \mathrm{P}<0.01$ vs. NCM460. CRC, colorectal cancer; $\mathrm{SNX} 3$, sorting nexin 3 .

In vivo metastasis. A total of 10 female nude mice (aged 4-5 weeks and weighing 16-22 g), purchased from Chengdu Dashuo Experimental Animal Co., Ltd, were maintained at $37^{\circ} \mathrm{C}$ and $50 \%$ humidity under a 12 -h light/dark cycle in an animal environmental control chamber with free access to food and water under specific pathogen-free conditions. HCT116 cells stably expressing SNX3 or vector control, were harvested from cell culture plates, washed with PBS, and resuspended at a concentration of $1 \times 10^{7}$ cells $/ \mathrm{ml}$ in PBS. A total of 10 mice were equally divided into two groups. Nude mice were injected with $1 \times 10^{6}$ cells in $100 \mu \mathrm{l}$ PBS in to the tail vein. After 6 weeks, these mice were sacrificed, and body weight was examined. All animal experiments were approved by the Animal Experimental Ethics Committee of The Third People's Hospital of Chengdu, and all procedures performed on animals were in accordance with the ethical standards of The Third People's Hospital of Chengdu.

Hematoxylin and eosin staining. Upon culling of the mice, the lungs were dissected and fixed with $4 \%$ paraformaldehyde for $30 \mathrm{~min}$ at room temperature, followed by embedding in paraffin. The tissues were sliced in to $5 \mu \mathrm{m}$ thick sections, and were stained with hematoxylin and eosin staining for $30 \mathrm{sec}$ at room temperature. The metastatic foci in the lungs were imaged and counted under a light microscope (magnification, x50 and x100, respectively).

Statistical analysis. SPSS 20.0 (IBM Corp.) and GraphPad Prism 5.0 (GraphPad Software, Inc.) were used for data analysis. Quantitative data are presented as the mean \pm standard error of the mean of three independent experiments. Comparisons between groups were analyzed using the unpaired two-tailed Student's t-test, or a one-way analysis of variance where appropriate with a Student's Newman-Keuls test for post-hoc analysis. $\mathrm{P}<0.05$ was considered to indicate a statistically significant difference.

\section{Results}

SNX3 expression is downregulated in human CRC cell lines. It has previously been reported that different CRC cell lines exhibit variable expression of Wnt protein (9). Considering the ability of SNX3 to mediate the secretion of $\mathrm{Wg}$, which is the Drosophila homolog of Wnt (7), the expression levels of SNX3 were examined in $11 \mathrm{CRC}$ cell lines and a human normal colon epithelial cell line. The results demonstrated that SNX3 expression was significantly decreased in the CRC cell lines compared with in the normal colon epithelial cell line NCM460 (Fig. 1A and B). These findings suggested that SNX3 expression may be downregulated in human CRC cell lines.

Expression of SNX3 is inversely associated with the migratory and invasive ability of CRC cells. To understand the role of SNX3 in CRC cells, the association between SNX3 expression and the migratory and invasive ability of CRC cells was evaluated. HCT116 and HT29 cells were used to investigate the effects of SNX3 on the migration and invasion of human CRC cells. HCT116 cells exhibited significantly increased migratory and invasive activity compared with HT29 cells (Fig. 2A-C). In addition, compared with HT29, the protein expression levels of vimentin were significantly upregulated, and E-cadherin was significantly downregulated in HCT116 (Fig. 2D and E). Furthermore, the expression levels of SNX3 were higher in HT29 cells compared with in HCT116 cells (Fig. 2D). These data indicated an inverse association between the expression of SNX3 and the invasive capacity of CRC cells.

SNX3 overexpression suppresses HCT116 migration and invasion. To determine whether SNX3 overexpression affects cellular processes that are involved in tumor metastasis, the effects of SNX3 overexpression on the migration and invasion of tumor cells was measured. Since HCT116 cells exhibited low SNX3 expression levels, a SNX3 lentivirus was transfected into these cells (Fig. 3A). Overexpression of SNX3 led to a decrease in the motility and invasiveness of HCT116 cells in vitro (Fig. $3 \mathrm{~B}$ and $\mathrm{C}$ ).

Silencing SNX3 promotes HT29 migration and invasion. To further evaluate the function of SNX3 on the migration and invasion of CRC cells, siRNAs were designed to knockdown SNX3 expression in HT29 cells. siRNA1 and siRNA2 were demonstrated to effectively knock down expression (Fig. 4A). siRNA2 was used in all subsequent experiments. Transwell assays demonstrated that SNX3 knockdown significantly increased the migratory and invasive ability of HT29 cells (Fig. 4B and C). 

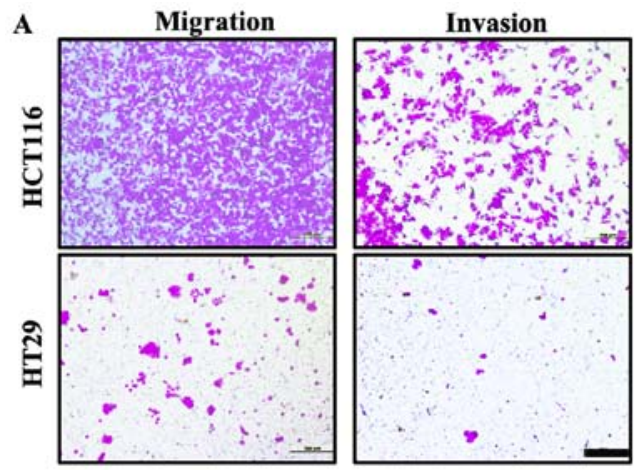

C
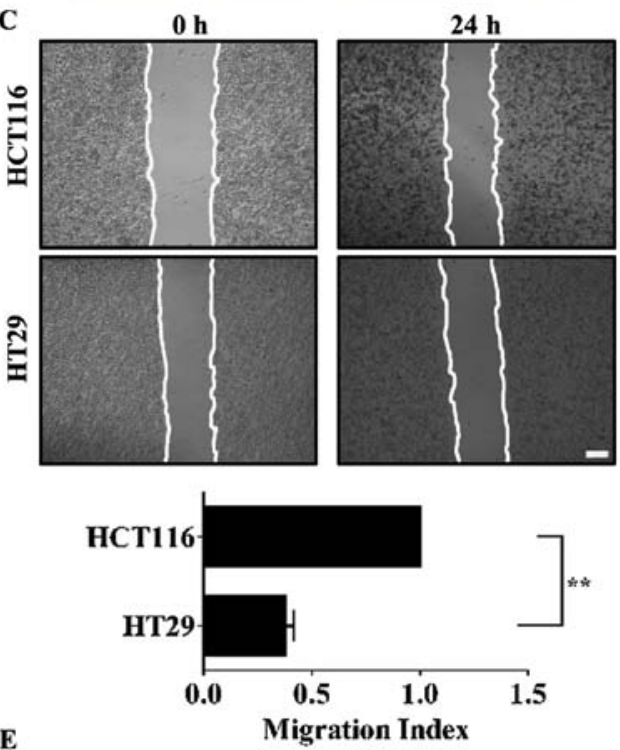

E

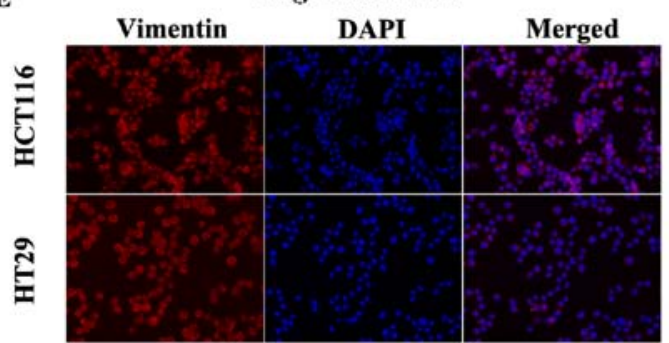

B
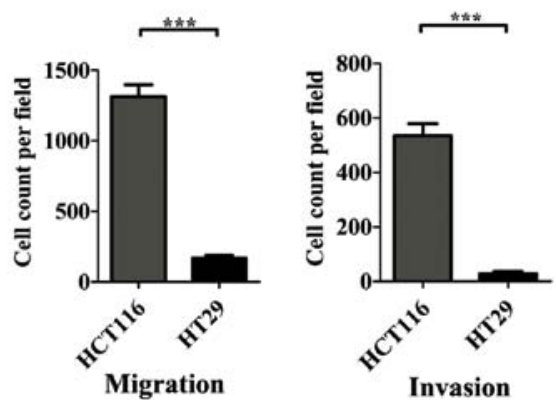

D
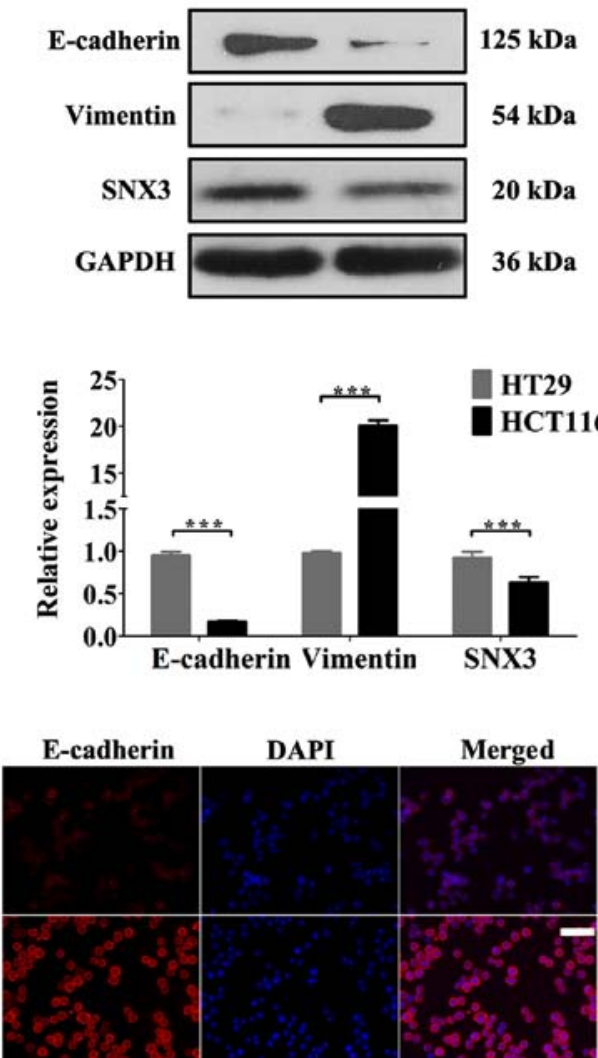

Figure 2. HT29 and HCT116 cells contain differential levels of SNX3, which is inversely associated with their migratory and invasive potential. (A) Transwell assays were performed to determine the migratory and invasive abilities of HT29 and HCT116 cells. Scale bar, $200 \mu \mathrm{m}$. (B) Number of cells that had invaded or migrated to the lower membrane surface in each field after $48 \mathrm{~h}$ was counted. (C) Representative images of the wound healing assay were obtained at 0 and $24 \mathrm{~h}$ after scratching. Scale bar, $50 \mu \mathrm{m}$. (D) Western blotting was performed to examine the expression levels of SNX3 and epithelial-mesenchymal transition-associated proteins in the colorectal cancer cell lines HCT116 and HT29. GAPDH was used as the loading control. (E) Protein expression levels of E-cadherin and vimentin were assessed by immunofluorescence in HT29 and HCT116 cells. Vimentin (red), E-cadherin (red) and DAPI (blue); scale bar, $50 \mu \mathrm{m} .{ }^{* *} \mathrm{P}<0.01 ;{ }^{* * *} \mathrm{P}<0.001$. SNX3, sorting nexin 3 .

SNX3 expression decreases expression of EMT-associated proteins. Since EMT is largely attributable to the migratory and invasive capacity of CRC cells (10), the expression levels of EMT markers were measured. To identify whether SNX3 inhibits EMT of HCT116 cells, the expression levels of EMT-associated proteins, E-cadherin and vimentin, which are considered markers of epithelial cells and mesenchymal cells, respectively, were determined. Overexpression of SNX3 increased the expression levels of E-cadherin and decreased the expression levels of vimentin (Fig. 3D and E). Conversely, the protein expression levels of E-cadherin were decreased, whereas vimentin expression was increased in HT29 cells following SNX3 knockdown (Fig. 4D and E). These findings suggested that SNX3 may be able to reverse EMT.
SNX3 may reverse EMT of CRC cells by regulating $\beta$-catenin. $\beta$-catenin signaling contributes to CRC pathogenesis and EMT (2). Based on assessment of the expression levels of $\beta$-catenin and SNX3 in HCT116 and HT29 cells, $\beta$-catenin may be negatively associated with SNX3 expression (Figs. 2D and 5A). Notably, SNX3 overexpression decreased the expression levels of $\beta$-catenin in HCT116 cells (Fig. 5A and B), whereas SNX3 knockdown in HT29 cells increased $\beta$-catenin expression (Fig. 5A-B). To examine the association between EMT and $\beta$-catenin, the EMT status and invasive ability of $\beta$-catenin-knockdown on HT29 cells transfected with SNX3 shRNA were examined. The results demonstrated that $\beta$-catenin knockdown in shRNA-SNX3-transfected HT29 cells significantly abrogated the EMT, migration and invasion induced by 
A
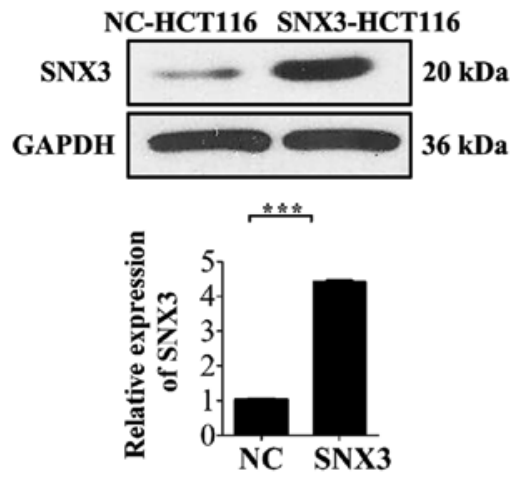

C

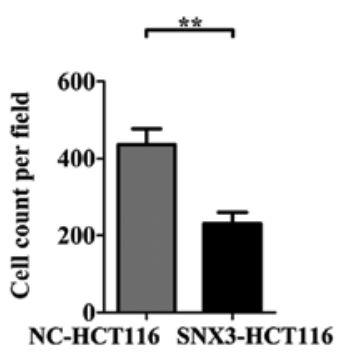

Migration
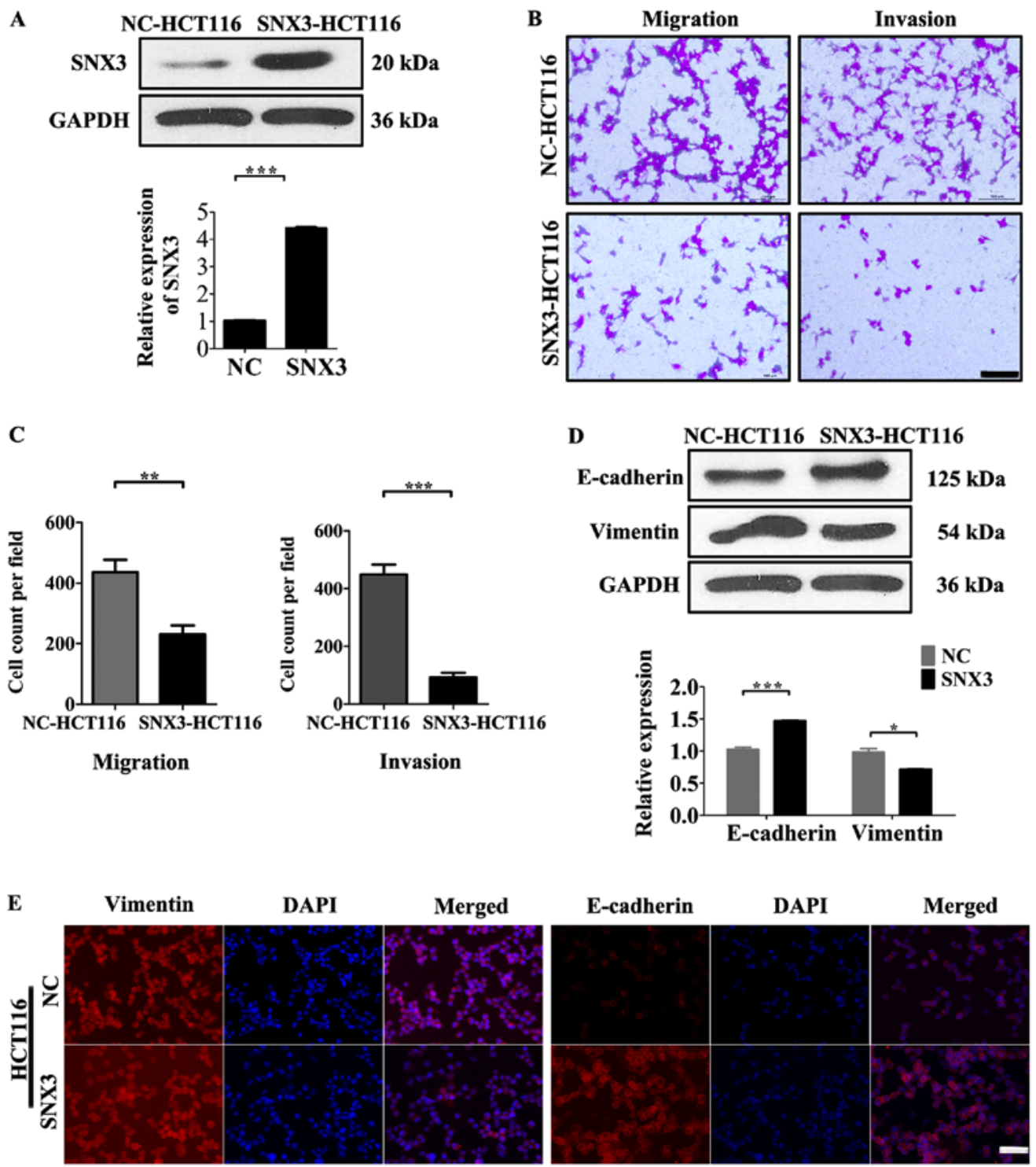

Figure 3. SNX3 inhibits the migration and invasion of HCT116 cells by partially reversing EMT. (A) Western blot analysis was conducted to determine SNX3 protein expression levels; GAPDH was used as the loading control. (B) Transwell assays were performed to determine the migratory and invasive abilities of NC-HCT116 and SNX3-HCT116 cells. Scale bar, $100 \mu \mathrm{m}$. (C) Number of cells that had invaded or migrated to the lower membrane surface in each field after $48 \mathrm{~h}$ was counted. (D) Western blotting was performed to examine the expression levels of SNX3 and EMT-associated proteins in NC-HCT116 and SNX3-HCT116 cells. GAPDH was used as the loading control. (E) Protein expression levels of E-cadherin and vimentin were detected by immunofluorescence in NC-HCT116 and SNX3-HCT116 cells. Vimentin (red), E-cadherin, (red) and DAPI (blue); scale bar, $50 \mu \mathrm{m} .{ }^{*} \mathrm{P}<0.05 ;{ }^{* *} \mathrm{P}<0.01 ;{ }^{* * *} \mathrm{P}<0.001 . \mathrm{EMT}$, epithelial-mesenchymal transition; NC, negative control; SNX3, sorting nexin 3.

SNX3 shRNA (Fig. 5D and E). Therefore, the results suggested that SNX3 may reverse EMT in CRC cells in a $\beta$-catenin dependent manner.

SNX3 inhibits the metastasis of CRC cells to the lung in vivo. To further investigate the role of SNX3 in lung metastasis of CRC cells in vivo, HCT116 cells were infected with SNX3 overexpression lentivirus or negative control to establish stable CRC cell lines. The effect of SNX3 on lung metastasis was assessed in immunodeficient female nude mice using tail vein injection. The degree of weight loss may be inversely associated with the severity of the lung metastasis. After 8 weeks, the weight of mice and the metastatic tumor nodules in the lungs were measured. The results demonstrated that the weight of mice was significantly decreased in mice injected with the negative control cells compared with those injected with
SNX3 overexpression cells (Fig. 6A). Additionally, a decreased number of total metastatic foci in the SNX3-overexpression group was observed (Fig. 6B and C). Hematoxylin and eosin staining was used to confirm the presence of metastatic tumor nodules in the lungs (Fig. 6D). These findings suggested that SNX3 may promote metastasis of CRC cells to the lungs.

\section{Discussion}

The most common cause of cancer-associated mortality is metastasis and EMT is the most common process by which tumorigenic cells become metastatic (11). Therefore, inhibiting this transformation may prove beneficial in cancer prevention. Wnt/ $\beta$-catenin signaling is considered to be the primary pathway that regulates EMT and the expression of EMT-associated molecules, including E-cadherin, vimentin 
A

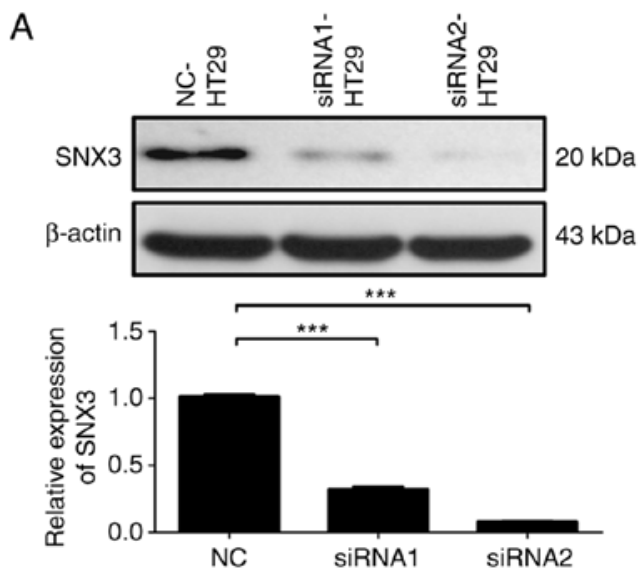

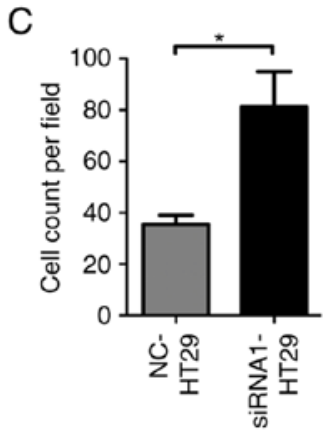

Migration

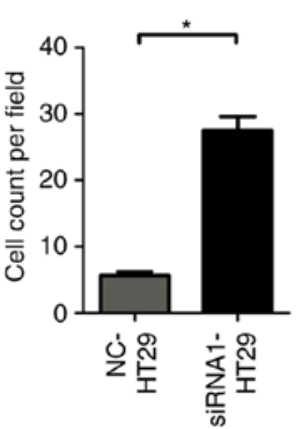

Invasion
B

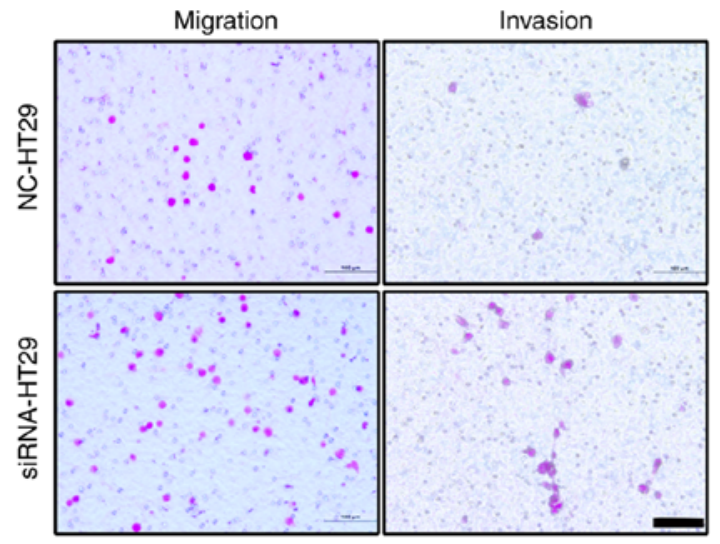

D
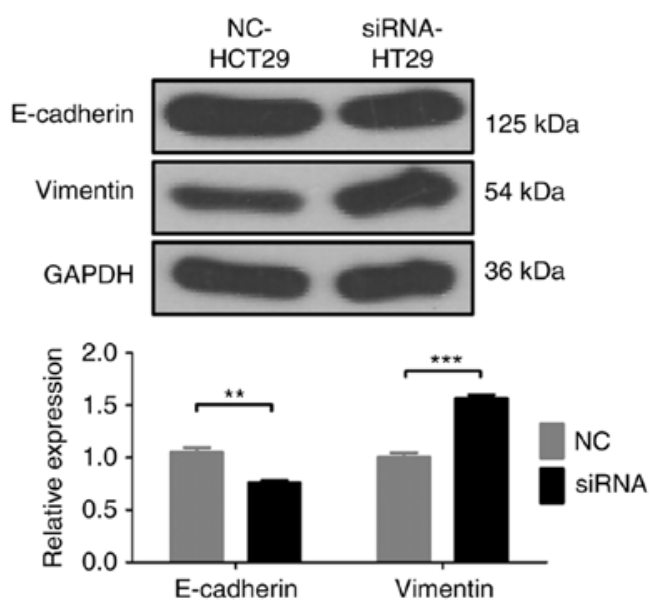

DAPI
Merged

E

Vimentin

DAPI

Merged
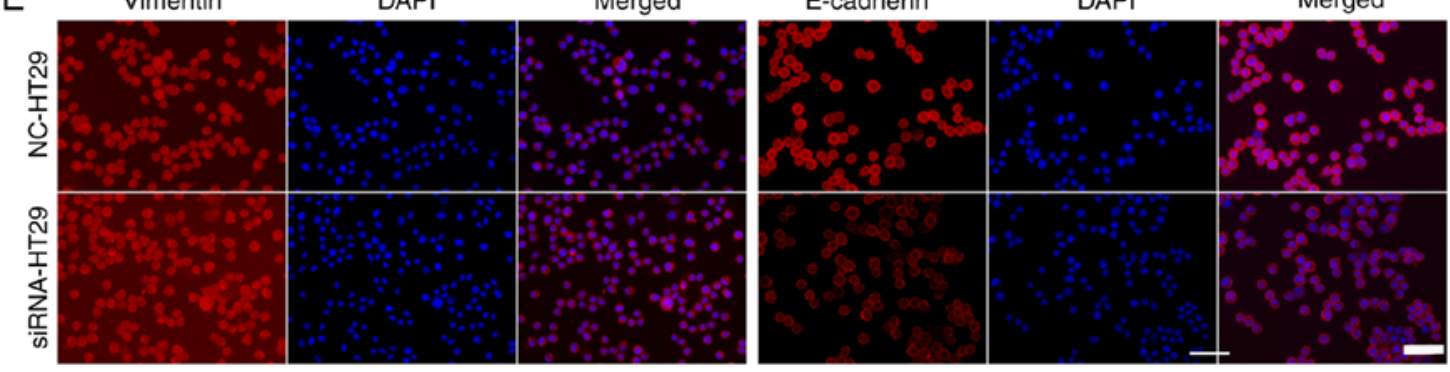

Figure 4. SNX3 knockdown promotes the migration and invasion of HT29 cells by promoting EMT. (A) Protein expression levels of SNX3 were detected by western blotting. GAPDH was used as the loading control. (B) Transwell assays were performed to determine the migratory and invasive abilities of NC-HT29 and si-SNX3-HT29 cells. Scale bar, $100 \mu \mathrm{m}$. (C) Number of cells that had invaded or migrated to the lower membrane surface in each field after $48 \mathrm{~h}$ was counted. (D) Western blotting was performed to examine the expression levels of EMT-associated proteins in NC-HT29 and si-SNX3-HT29 cells. GAPDH was used as the loading control. (E) Protein expression levels of E-cadherin and vimentin were detected by immunofluorescence in NC-HT29 and si-SNX3-HT29 cells. Vimentin (red), E-cadherin (red) and DAPI (blue); scale bar, $50 \mu \mathrm{m} .{ }^{*} \mathrm{P}<0.05 ;{ }^{* *} \mathrm{P}<0.01 ;{ }^{* * *} \mathrm{P}<0.001$. EMT, epithelial-mesenchymal transition; NC, negative control; si/siRNA, small interfering RNA; SNX3, sorting nexin 3.

and Snail $(12,13)$. Therefore, suppression of the Wnt/ $\beta$-catenin signaling pathway and reversal of EMT in cancer cells may be a potential approach to cancer therapy (3). In the present study, the results suggested that SNX3 may reverse EMT, and inhibit the migratory and invasive potential of CRC cells. In addition, it was demonstrated that SNX3 was differentially expressed in CRC cells with different metastatic capacities. When SNX3 was overexpressed in HCT116 cells, the typically high migratory and invasive potential of the cells was suppressed, and the expression of EMT-associated proteins were decreased. the mesenchymal phenotype reverted back to that of epithelial cells. Conversely, HT29 cells exhibited an increase in migratory and invasive capacity following knockdown of SNX3. SNX3 is a transporter for the secretion of Wnt proteins; therefore, it was hypothesized that the actions of SNX3 may be associated with $\beta$-catenin signaling. $\beta$-catenin was negatively associated with SNX3 expression, and knockdown of $\beta$-catenin expression reduced migration and invasion. Furthermore, SNX3 overexpression prevented lung metastasis of injected HCT116 cells in vivo.

Sorting nexins are part of a large, evolutionarily ancient family of proteins in mammals. These proteins regulate the trafficking of various proteins among intracellular membranes, including endocytosis, protein degradation and recycling (14). SNX3 is the only sorting nexin that mediates secretion of Wnt protein and retrieval of its receptor, 
A

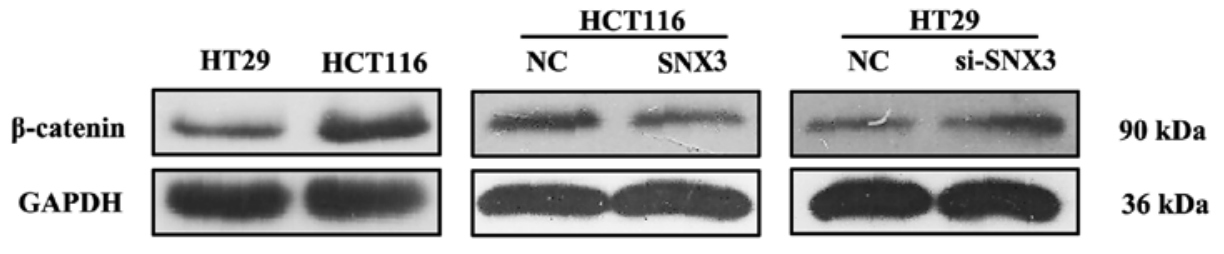

B
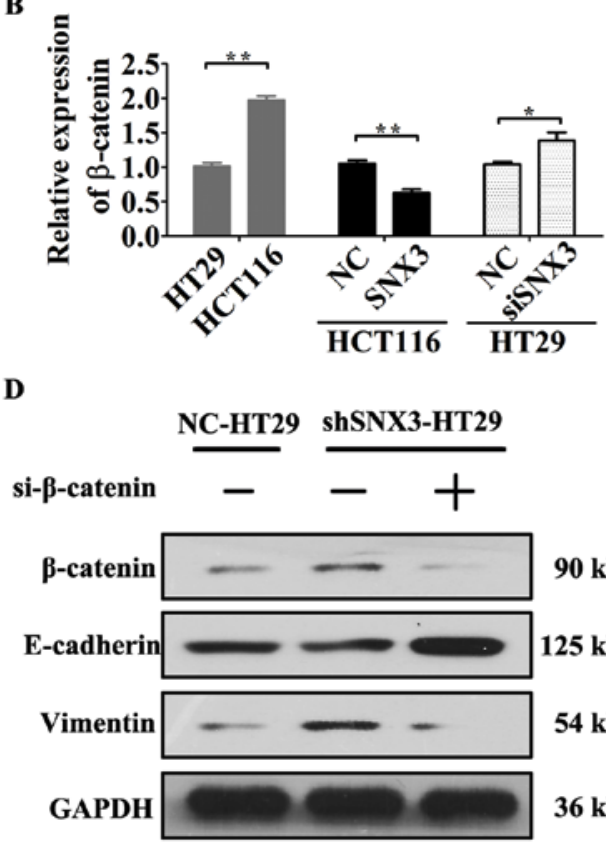

C

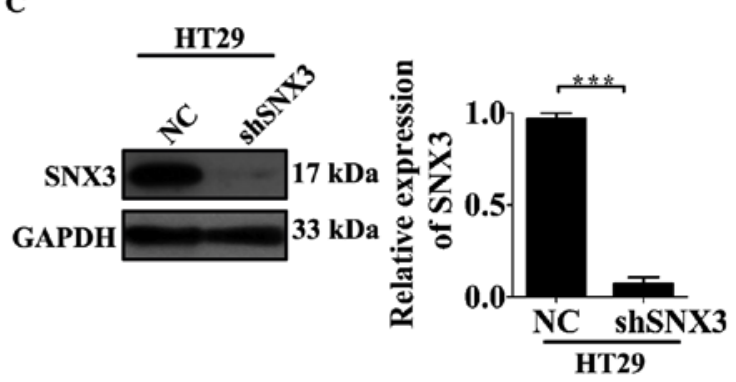

shSNX3+si- $\beta$-catenin

$90 \mathrm{kDa}$

$125 \mathrm{kDa}$

$54 \mathrm{kDa}$

$36 \mathrm{kDa}$
NC

$\operatorname{shSNX3}$

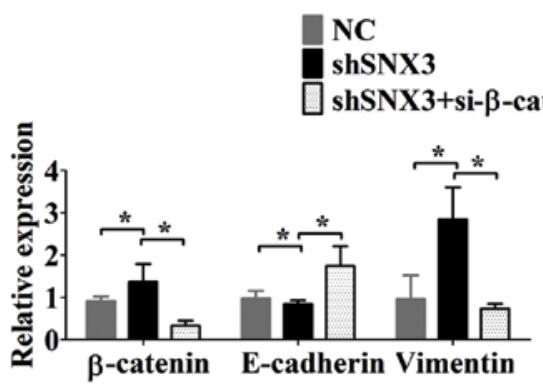

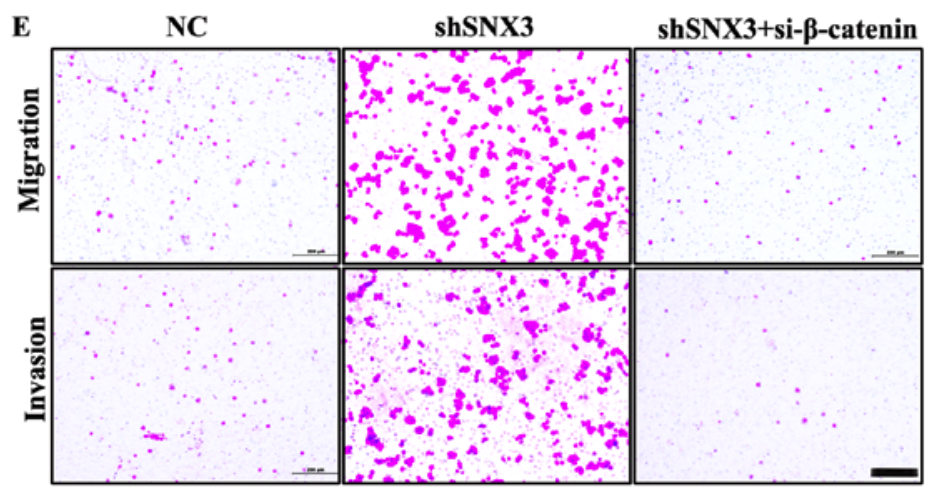

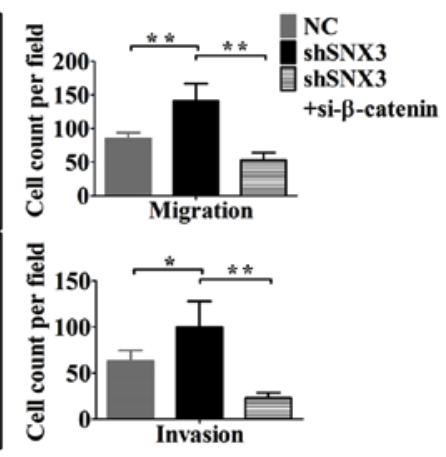

Figure 5. SNX3 may reverse EMT of CRC cells by regulating $\beta$-catenin. (A) Protein expression levels of $\beta$-catenin were determined by western blotting. (B) $\beta$-catenin expression was semi-quantified. (C) Following transfection with sh-SNX3, the expression levels of $\beta$-catenin were detected by western blotting. (D) Protein expression levels of $\beta$-catenin and EMT-associated proteins in NC-HT29, shSNX3-HT29 and shSNX3-HT29-si- $\beta$-catenin cells were analyzed by western blotting with GAPDH as the loading control. (E) Transwell assays were performed to determine the migratory and invasive abilities of NC-HT29, shSNX3-HT29 and shSNX3-HT29-si- $\beta$-catenin cells; scale bar, $200 \mu \mathrm{m} .{ }^{*} \mathrm{P}<0.05 ;{ }^{* *} \mathrm{P}<0.01 ;{ }^{* * * *} \mathrm{P}<0.001$. EMT, epithelial-mesenchymal transition; NC, negative control; sh, small hairpin RNA; si, small interfering RNA; SNX3, sorting nexin 3.

Wntless (6,7). Although SNX3 is important for Wnt signaling and epidermal growth factor receptor trafficking (15), information on its role in cancer prevention is limited. The present study demonstrated that SNX3 may reverse the EMT in CRC cells by suppressing $\beta$-catenin, thus inhibiting metastasis. These findings provide novel insights into the functions of SNX3, particularly in regulating the EMT and cancer metastasis.

At least $19 \mathrm{Wnt}$ members have been identified to date. Various Wnt proteins initiate canonical or noncanonical $\beta$-catenin signals for distinctive biological purposes $(16,17)$. For example, in CRC, the secretion of Wnt3a, a common canonical Wnt, acts as an oncogene that activates $\beta$-catenin (5); whereas, a noncanonical Wnt, Wnt5a, serves as a tumor suppressor that impairs invasion, migration and metastasis of CRC cells (18). Therefore, two possible mechanisms by which SNX3 inhibits $\beta$-catenin are hypothesized. Firstly, SNX3 may enhance non-canonical Wnt signaling. Notably, Wnt5a enhances $\beta$-catenin degradation, downregulates its expression in CRC cells (19), and is associated with a good prognosis for patients with CRC (18). Secondly, SNX3 may affect the stability of Wnt receptors, through a similar mechanism to that of SNX27, which enhances the endocytosis of Fzd7 and promotes the degradation of Fzd7, thus repressing the signals transduced by $\beta$-catenin (20). In the present study, examining the secretion of Wnt 3 or Wnt5a by HT29 cells overexpressing 

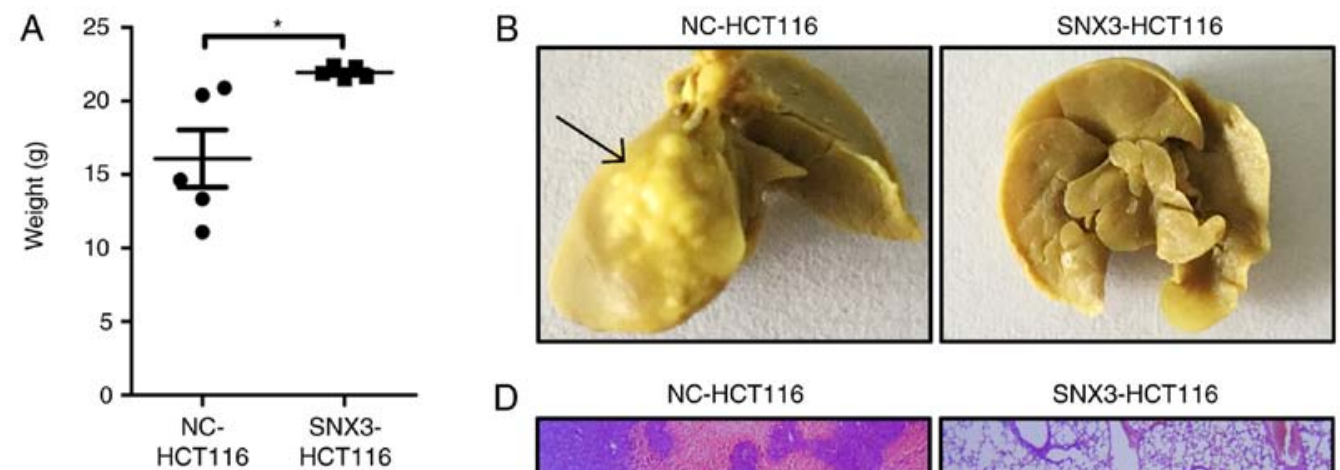

D
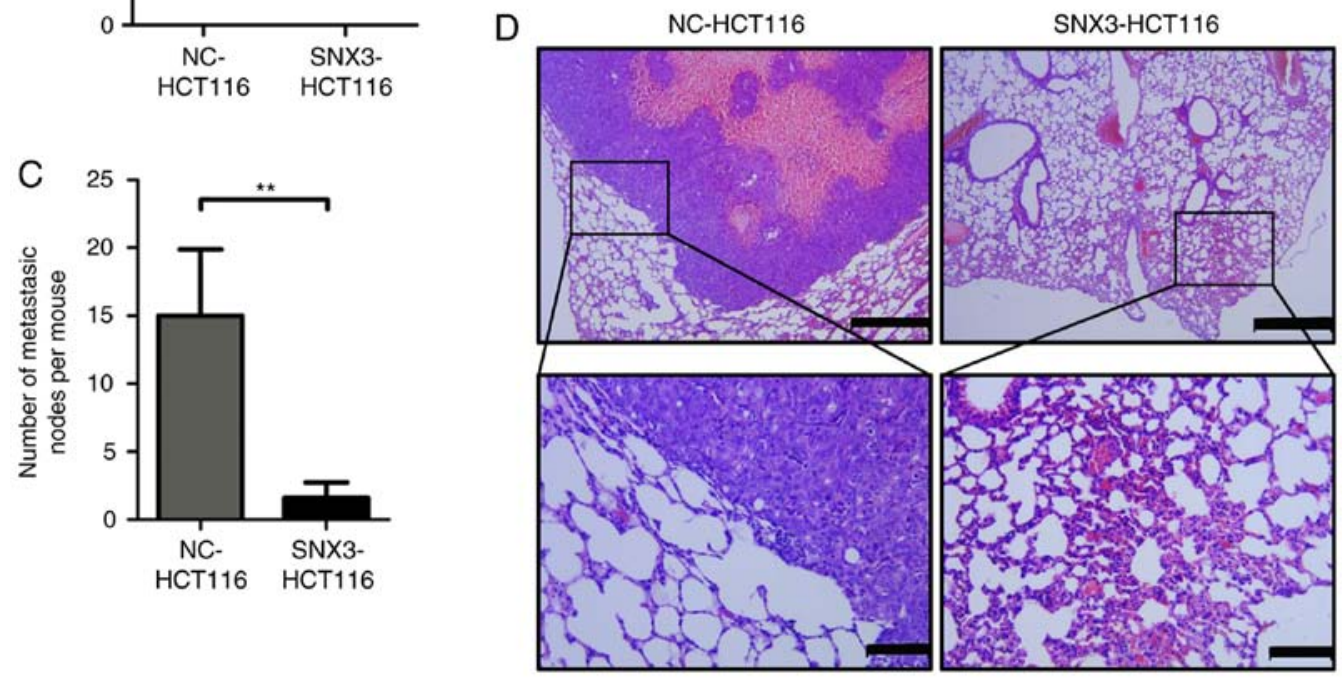

Figure 6. SNX3 inhibits the metastasis of colorectal cancer cells in a xenograft mouse model. (A) Nude mice were intravenously injected with NC-HCT116 or SNX3-HCT116 cells. The average body weight of nude mice was measured. (B) Representative images of the lungs dissected from nude mice are presented (black arrows show the metastatic nodes). Upper scale bars, $500 \mu \mathrm{m}$. Lower scale bars, $200 \mu \mathrm{m}$. (C) Number of metastatic nodes was measured. (D) Hematoxylin and eosin staining of lung tissue images of representative mice from the different experimental groups 8 weeks after injection. ${ }^{*} \mathrm{P}<0.05 ;{ }^{* *} \mathrm{P}<0.01$. NC, negative control; SNX3, sorting nexin 3.

SNX3 was attempted; however, in the supernatant of cultured CRC cells, the quantity of secretory Wnt was too low to be effectively detected (data not shown). Future studies need to further investigate the effects of SNX3 on CRC.

\section{Acknowledgements}

Not applicable.

\section{Funding}

This study was supported by grants from The National Natural Science Foundation of China (grant no. 81270465 to $\mathrm{YG}$ ), the Natural Science Foundation of Sichuan Province (grant nos. 2015FZ0072 to YG and 2014JY0017 to $\mathrm{CZ}$ ) and the Science and Technology Foundation of Chengdu City (grant nos. 2014-HM01-00217-SF to CZ and 2015-HM01-00139-SF to ZZ).

\section{Availability of data and material}

The datasets used and/or analyzed during the current study are available from the corresponding author on reasonable request.

\section{Authors' contributions}

BRP, CZ and YBG were responsible for the study design, original article drafting and editing, data acquisition and data analysis.
BRP, TTZ, WY, YJL, ZZ, YT, YNC, JWZ and YLL performed the experiments. BRP performed the tumor xenograft experiments. All authors have read and approved the final manuscript.

\section{Ethics approval and consent to participate}

All animal experiments were approved by the Animal Experimental Ethics Committee of the Third People's Hospital of Chengdu, and all procedures performed on animals were in accordance with the ethical standards of the Third People's Hospital of Chengdu.

\section{Patient consent for publication}

Not applicable.

\section{Competing interests}

The authors declare that they have no competing interests.

\section{References}

1. GBD 2013 Mortality and Causes of Death Collaborators: Global, regional, and national age-sex specific all-cause and cause-specific mortality for 240 causes of death, 1990-2013: A systematic analysis for the Global Burden of Disease Study 2013. Lancet 385: 117-171, 2015.

2. White BD, Chien AJ and Dawson DW: Dysregulation of Wnt $/ \beta$-catenin signaling in gastrointestinal cancers. Gastroenterology 142: 219-232, 2012 . 
3. Masuda M, Sawa $M$ and Yamada T: Therapeutic targets in the Wnt signaling pathway: Feasibility of targeting TNIK in colorectal cancer. Pharmacol Ther 156: 1-9, 2015.

4. Schwab RHM, Amin N, Flanagan DJ, Johanson TM, Phesse TJ and Vincan E: Wnt is necessary for mesenchymal to epithelial transition in colorectal cancer cells. Dev Dyn 247: 521-530, 2018.

5. Voloshanenko O, Erdmann G, Dubash TD, Augustin I, Metzig M, Moffa G, Hundsrucker C, Kerr G, Sandmann T, Anchang B, et al: Wnt secretion is required to maintain high levels of Wnt activity in colon cancer cells. Nat Commun 4: 2610, 2013.

6. Harterink M, Port F, Lorenowicz MJ, McGough IJ, Silhankova M, Betist MC, van Weering JRT, van Heesbeen RGHP, Middelkoop TC, Basler K, et al: A SNX3-dependent retromer pathway mediates retrograde transport of the Wnt sorting receptor Wntless and is required for Wnt secretion. Nat Cell Biol 13: 914-923, 2011.

7. Zhang P, Wu Y, Belenkaya TY and Lin X: SNX3 controls Wingless/Wnt secretion through regulating retromer-dependent recycling of Wntless. Cell Res 21: 1677-1690, 2011.

8. Thompson CC, Ashcroft FJ, Patel S, Saraga G, Vimalachandran D, Prime W, Campbell F, Dodson A, Jenkins RE, Lemoine NR, et al: Pancreatic cancer cells overexpress gelsolin family-capping proteins, which contribute to their cell motility. Gut 56: 95-106, 2007.

9. Holloway KR, Calhoun TN, Saxena M, Metoyer CF, Kandler EF, Rivera CA and Pruitt K: SIRT1 regulates Dishevelled proteins and promotes transient and constitutive Wnt signaling. Proc Natl Acad Sci USA 107: 9216-9221, 2010.

10. Zhu QC, Gao RY, Wu W and Qin HL: Epithelial-mesenchymal transition and its role in the pathogenesis of colorectal cancer. Asian Pac J Cancer Prev 14: 2689-2698, 2013.

11. Hanahan D and Weinberg RA: Hallmarks of cancer: The next generation. Cell 144: 646-674, 2011.

12. Huber AH and Weis WI: The structure of the beta-catenin/ E-cadherin complex and the molecular basis of diverse ligand recognition by beta-catenin. Cell 105: 391-402, 2001.
13. Gilles C, Polette M, Mestdagt M, Nawrocki-Raby B, Ruggeri P, Birembaut $\mathrm{P}$ and Foidart JM: Transactivation of vimentin by beta-catenin in human breast cancer cells. Cancer Res 63: 2658-2664, 2003.

14. Cullen PJ and Korswagen HC: Sorting nexins provide diversity for retromer-dependent trafficking events. Nat Cell Biol 14: 29-37, 2012.

15. Chiow KH, Tan Y, Chua RY, Huang D, Ng ML, Torta F, Wenk MR and Wong SH: SNX3-dependent regulation of epidermal growth factor receptor (EGFR) trafficking and degradation by aspirin in epidermoid carcinoma (A-431) cells. Cell Mol life Sci 69: 1505-1521, 2012.

16. Lee JM,Kim IS, Kim H,Lee JS, Kim K, Yim HY, Jeong J, Kim JH, Kim JY, Lee H, et al: RORalpha attenuates Wnt/beta-catenin signaling by PKCalpha-dependent phosphorylation in colon cancer. Mol Cell 37: 183-195, 2010.

17. Kikuchi A, Yamamoto $\mathrm{H}$ and Sato A: Selective activation mechanisms of Wnt signaling pathways. Trends Cell Biol 19: 119-129, 2009.

18. Dejmek J, Dejmek A, Säfholm A, Sjölander A and Andersson T: Wnt-5a protein expression in primary dukes B colon cancers identifies a subgroup of patients with good prognosis. Cancer Res 65: 9142-9146, 2005.

19. Ying J, Li H, Yu J, Ng KM, Poon FF, Wong SC, Chan AT, Sung JJ and Tao Q: WNT5A exhibits tumor-suppressive activity through antagonizing the Wnt/beta-catenin signaling, and is frequently methylated in colorectal cancer. Clin Cancer Res 14: 55-61, 2008

20. Sun L, Hu X, Chen W, He W, Zhang Z and Wang T: Sorting nexin 27 interacts with Fzd7 and mediates Wnt signalling. Biosci Rep 36: e00296, 2016.

This work is licensed under a Creative Commons Attribution-NonCommercial-NoDerivatives 4.0 International (CC BY-NC-ND 4.0) License. 